\title{
Outcome of COVID-19 infection in 50 multiple myeloma patients treated with novel drugs: single-center experience
}

\author{
Marta Krejci $^{1}$ (1) Ludek Pour $^{1} \cdot$ Zdenek Adam $^{1} \cdot$ Viera Sandecka $^{1} \cdot$ Martin Stork $^{1} \cdot$ Sabina Sevcikova ${ }^{2} \cdot$ Martin Krejci $^{1}$. \\ Zdenka Knechtova $^{1} \cdot$ Zdenek Kral $^{1}$
}

Received: 27 April 2021 / Accepted: 22 June 2021 / Published online: 26 July 2021

(c) The Author(s), under exclusive licence to Springer-Verlag GmbH Germany, part of Springer Nature 2021

\begin{abstract}
Infections are the primary cause of morbidity and mortality in multiple myeloma (MM) patients (pts). The aim of our retrospective analysis was to evaluate incidence and course of COVID-19 infection in a cohort of $351 \mathrm{MM}$ outpatients treated with novel drugs. COVID-19 disease was detected in 50/351 pts (14\%); median age was 68 years. Gender, ISS stage, and last treatment lines were as follows: male 32, female 18; ISS-I 19, ISS-II 20, ISS-III 11; daratumumab-based 15, lenalidomide-based 12, bortezomib-based 17, other 6. Positive PCR test at COVID-19 diagnosis was present in all pts; anti-myeloma treatment was interrupted. Hospitalizations for COVID-19 pneumonia were necessary for 28/50 pts (56\%), 18/50 pts (36\%) in standard unit (SU) $10 / 50$ pts (20\%) in intensive care unit (ICU), and 9/50 pts (18\%) died. The statistically significant parameters for COVID-19 hospitalization were as follows: responsive versus non-responsive disease $(p=0.027)$, ECOG performance status $0-2$ versus $\geq 3(p=0.014)$, presence of comorbidities $(0-1$ versus $\geq 2, p=0.043)$. The statistically significant factors for COVID-19 death were as follows: ECOG $0-2$ versus $\geq 3(p=0.001)$, presence of comorbidities $(0-1$ versus $\geq 2, p=0.007)$, serious course of COVID-19 disease with ICU hospitalization (SU versus ICU, $p=0.001$ ). None of the other studied risk factors was associated with poor outcome (age, gender, ISS stage, immunoparesis, type of anti-myeloma treatment). Full recovery from COVID-19 infection was observed in 41/50 pts (82\%) in median of 32 days. The course of COVID-19 disease in MM pts was mostly moderate or serious with $56 \%$ of hospitalizations and $18 \%$ of deaths.
\end{abstract}

Keywords Multiple myeloma $\cdot$ COVID-19 $\cdot$ Novel drugs $\cdot$ Hospitalization $\cdot$ Mortality

\section{Introduction}

The coronavirus disease 2019 (COVID-19), caused by the coronavirus SARS-Cov-2, has become a global pandemic since its first occurrence in late 2019 [1]. Clinical manifestation of COVID 19 is very variable, from patients (pts) with mild, mostly respiratory symptoms to pts with severe COVID pneumonia and multi-organ failure [2]. Reported risk factors for severe COVID-19 disease are predominantly

Marta Krejci

krejci.marta@fnbrno.cz

1 Department of Internal Medicine, Hematology and Oncology, Faculty of Medicine, University Hospital Brno and Masaryk University, Jihlavska 20, Brno 625 00, Czech Republic

2 Babak Myeloma Group, Department of Pathophysiology, Faculty of Medicine, Masaryk University, Brno,

Czech Republic advanced age and various comorbidities [3]. Cancer patients are at higher risk of developing a severe form of COVID-19 [4].

Optimal treatment for COVID 19 is not yet clear; various treatment options have been published with different results. Currently, there is no standard approved therapy for COVID-19; mostly supportive care has been often used for COVID-19 pts [5]. Some studies reported remdesivir as well as treatment with convalescent plasma as beneficial treatment possibilities for pts hospitalized for COVID-19 [6, 7].

Multiple myeloma (MM) is a hematological malignancy involving plasma cells; MM pts have an inherently compromised humoral and cellular immunity $[8,9]$. Pathogenesis of MM results in the suppression of the adaptive immune system and leads to low levels of immunoglobulin production. Immunoparesis (hypogammaglobulinemia) is very frequent; it is present in $85-90 \%$ of MM pts [10, 11]. Correlation of immunoparesis and increased risk of adverse COVID-19 outcome is unclear. 
The introduction of new therapeutic agents, such as immunomodulatory drugs (IMIDs), proteasome inhibitors (PI), and monoclonal antibodies, has resulted in significant progress in the treatment of MM with increased survival rates [12]. However, several of these novel treatments in combination with corticosteroids are associated with an increased risk of infections with substantial hematological toxicity, including neutropenia and lymphopenia [13-15].

The aims of our retrospective analysis were to evaluate incidence and course of COVID-19 infection and clinical outcome in a cohort of $351 \mathrm{MM}$ pts treated with novel drugs in one institution and to define the possible significant laboratory and clinical prognostic parameters for adverse outcome of COVID-19-associated pneumonia, necessity of hospital admission for COVID-19 or death for COVID-19 in these immunocompromised MM pts.

\section{Patients and methods}

\section{Incidence of COVID-19 disease and immunoparesis in $351 \mathrm{MM}$ patients treated with novel drugs}

From October 2020 to February 2021, 351 (100\%) symptomatic MM pts were treated at the outpatient clinic of the Department of Internal Medicine, Hematology and Oncology, University Hospital Brno, Czech Republic, with various combinations of novel drugs. All patients signed the informed consent form approved by the Ethics board of the hospital before start of MM treatment. COVID-19 infection was detected in 50/351 pts (14\%); all these pts were SARSCov-2 positive as confirmed by either PCR test or antigen test from a nasopharyngeal swab. All patients with suspected COVID-19 pneumonia received computer tomography (CT) examination of thorax. Immunoparesis (reduction below the lower normal limit of one or two uninvolved immunoglobulins- $\operatorname{IgG}<6.7 \mathrm{~g} / \mathrm{L}, \operatorname{IgA}<0.88 \mathrm{~g} / \mathrm{L}, \operatorname{IgM}<0.27 \mathrm{~g} / \mathrm{L}$ ) was presented in $85 \%$ (298/351) of pts at the start of anti-myeloma treatment. There were no significant differences in incidence of immunoparesis between COVID-19-infected pts (43/50, $87 \%)$ and COVID-19-non-infected pts (255/301, 85\%).

\section{Treatment for multiple myeloma in $50 \mathrm{MM}$ pts with COVID-19 disease}

There were four types of anti-myeloma treatment: daratumumab-based therapy (15 pts, 30\%), bortezomib-based therapy without daratumumab (17 pts, 34\%), lenalidomide-based treatment without daratumumab (12 pts, 24\%), other type therapy with novel drugs, such as carfilzomib or pomalidomide (6 pts, 12\%). All MM treatments included corticosteroids as a part of treatment (dexamethasone or prednisone). MM staging was carried out according to the
International Staging System (ISS) [16]. Standard criteria were used for the evaluation of MM response [17]. Upon COVID-19 symptom onset, MM treatment was interrupted in all cases.

\section{Clinical manifestation of COVID-19 disease}

The clinical manifestation of COVID-19 disease was variable, from mild symptoms of upper respiratory infection without fever treated fully ambulatory to COVID-19 pneumonia with dry cough, dyspnea, fever, and hypoxia and in some cases with respiratory failure. Other non-specific symptoms were arthralgia, myalgia, fatigue, headaches, and diarrhea. All our MM pts with COVID-19 pneumonia (28/50 cases, 54\%) were hospitalized. We recognized three types of COVID-19 infection in our set of pts: mild course, treated fully ambulatory, moderate course with hospitalization at standard unit (SU), and severe course with hospitalization at intensive care unit (ICU). No previous anti-COVID vaccination was done in this cohort of pts.

\section{Laboratory and clinical parameters and their possible relationship to COVID-19 outcome}

We retrospectively analyzed the main clinical characteristics of MM pts at the time of COVID-19 infection, such as age, gender, ISS stage, type of monoclonal immunoglobulin (MIg), comorbidities, presence of immunoparesis, performance status according to ECOG grade [18], number of myeloma treatment lines, response to the last treatment line, type of treatment line with various novel drugs, and other with aim to find possible relationship to COVID-19 outcome.

\section{Statistics}

Descriptive statistics for demographic characteristics and clinical variables were used. Differences between categorical variables were analyzed with Fisher's exact test. A two-sided $p$ value of $\leq 0.05$ was considered significant. All statistical analyses were performed using IBM ${ }^{\circledR S P S S}{ }^{\circledR}$ Statistics Version 27.

\section{Results}

\section{Patient characteristics}

Characteristics of cohort $50 \mathrm{MM}$ pts on anti-myeloma therapy with novel drugs, who suffered from COVID-19 infection, are summarized in Table 1. Median age at time of COVID-19 infection was 68 years (range 41-86). Thirty-two pts $(64 \%)$ were male; 18 pts (36\%) were female (Table 1$)$. 
Table 1 Patient characteristics: cohort of $50 \mathrm{MM}$ pts with COVID-19 infection

\begin{tabular}{|c|c|c|c|c|}
\hline Parameter-type & Parameter-subtype & $\begin{array}{l}\text { All pts: } \\
\text { Number }(\%) \\
50(100)\end{array}$ & $\begin{array}{l}\text { Ambulatory pts: } \\
\text { Number }(\%) \\
22(100)\end{array}$ & $\begin{array}{l}\text { Hospitalized pts: } \\
\text { Number }(\%) \\
28(100)\end{array}$ \\
\hline Age in years (median; range) & & $68 ; 41-86$ & $66 ; 41-81$ & $69 ; 55-86$ \\
\hline Gender & $\begin{array}{l}\text { Female } \\
\text { Male }\end{array}$ & $\begin{array}{l}18(36) \\
32(64))\end{array}$ & $\begin{array}{l}7(32) \\
15(68)\end{array}$ & $\begin{array}{l}11(39) \\
17(61)\end{array}$ \\
\hline ISS stage & $\begin{array}{l}\text { ISS-I and II } \\
\text { ISS-III }\end{array}$ & $\begin{array}{l}39(78) \\
11(22)\end{array}$ & $\begin{array}{l}16(73) \\
6(27)\end{array}$ & $\begin{array}{l}23(82) \\
5(18)\end{array}$ \\
\hline Type of M-protein & $\begin{array}{l}\text { IgG } \\
\text { IgA } \\
\text { Light chain }\end{array}$ & $\begin{array}{l}39(78) \\
8(16) \\
3(6)\end{array}$ & $\begin{array}{l}16(72) \\
5(23) \\
1(5)\end{array}$ & $\begin{array}{l}20(71) \\
6(21) \\
2(8)\end{array}$ \\
\hline Line of MM treatments & $\begin{array}{l}1 \text { or } 2 \\
\geq 3\end{array}$ & $\begin{array}{l}37(74) \\
13(26)\end{array}$ & $\begin{array}{l}19(86) \\
3(14)\end{array}$ & $\begin{array}{l}18(64) \\
10(36)\end{array}$ \\
\hline ECOG grade & $\begin{array}{l}0-2 \\
\geq 3\end{array}$ & $\begin{array}{l}40(80) \\
10(20)\end{array}$ & $\begin{array}{l}21(95) \\
1(5)\end{array}$ & $\begin{array}{l}18(64) \\
10(36)\end{array}$ \\
\hline Comorbidities & $\begin{array}{l}0 \text { or } 1 \\
\geq 2\end{array}$ & $\begin{array}{l}20(40) \\
30(60)\end{array}$ & $\begin{array}{l}12(55) \\
10(45)\end{array}$ & $\begin{array}{l}7(25) \\
21(75)\end{array}$ \\
\hline Last MM treatment before COVID-19 infection & $\begin{array}{l}\text { Daratumumab-based } \\
\text { Lenalidomide-based } \\
\text { Bortezomib-based } \\
\text { Other }\end{array}$ & $\begin{array}{l}15(30) \\
12(24) \\
17(34) \\
6(12)\end{array}$ & $\begin{array}{l}4(22) \\
8(36) \\
8(36) \\
2(6)\end{array}$ & $\begin{array}{l}11(39) \\
4(14) \\
9(33) \\
4(14)\end{array}$ \\
\hline Disease status at COVID-19 infection & $\begin{array}{l}\text { Responsive disease } \\
\text { Active disease }\end{array}$ & $\begin{array}{l}33(69) \\
15(31)\end{array}$ & $\begin{array}{l}19(86) \\
3(14)\end{array}$ & $\begin{array}{l}15(54) \\
13(46)\end{array}$ \\
\hline
\end{tabular}

Concomitant cardiovascular or pulmonary comorbidities were seen in 46 pts (96\%), of which hypertension was the most common (35 pts). Some MM pts had 2 or more comorbidities (30 pts, 60\%), such as chronic ischemic heart disease, diabetes mellitus, chronic obstructive pulmonary disease, and others. In our cohort, there were $34 \mathrm{MM}$ pts $(68 \%)$ in remission of disease (complete remission (CR), very good partial remission (VGPR), or partial remission (PR)) and $16 \mathrm{MM}$ pts (32\%) with non-responsive disease (minimal response (MR), stable disease (SD), or progression of disease (PD)).

The laboratory findings included blood count level and value of $\mathrm{C}$-reactive protein and creatinine were available in 32 pts $(32 / 50,64 \%)$ (summarized in Table 2). The analysis of COVID-19-specific antibodies was not done in our group of pts. There were two main types of COVID course in our pts, fully ambulatory course (22 pts, $44 \%$ ) and course with hospitalization (28 pts, $56 \%$ ).

\section{Outcome and therapy of COVID-19 infection in MM patients}

The COVID-19 treatment in $22 \mathrm{MM}$ pts (22/50, 44\%) with ambulatory course of COVID-19 was mostly symptomatic and supportive care with prophylactic use of antibiotics to prevent bacterial superinfection in pts with CRP elevation. All these MM pts recovered completely in median of 22 days
Table 2 Laboratory values at diagnosis of COVID-19 infection in 33 multiple myeloma patients

\begin{tabular}{lcc}
\hline Parameter & Median; range & $\begin{array}{l}\text { Normal } \\
\text { range of } \\
\text { values }\end{array}$ \\
\hline $\mathrm{CRP}, \mathrm{mg} / \mathrm{L}$ & $14 ; 1-300$ & $0-5$ \\
Hemoglobin, g/L & $105 ; 84-154$ & $130-170$ \\
Leukocytes, $10^{9} / \mathrm{L}$ & $4.7 ; 2.13-11.3$ & $4-10$ \\
Neutrophils, $10^{9} / \mathrm{L}$ & $2.75 ; 0.82-9.37$ & $2-7$ \\
Lymphocytes, $10^{9} / \mathrm{L}$ & $0.795 ; 0.18-4.09$ & $0.8-4$ \\
Thrombocytes, $10^{9} / \mathrm{L}$ & $149 ; 2-399$ & $150-400$ \\
Creatinine, $\mu \mathrm{mol} / \mathrm{L}$ & $94 ; 63-540$ & $60-100$ \\
\hline
\end{tabular}

(range 20-45); after this, they continued in their anti-myeloma treatment.

In total, $28 \mathrm{MM}$ pts $(28 / 50,56 \%)$ were admitted due to COVID-19 pneumonia. Hospitalization in SD was necessary in 18 pts (18/28, 64\% from all COVID-19 hospitalizations); 10 pts $(10 / 28,36 \%)$ were admitted in ICU. Invasive pulmonary ventilation (IPV) was done in $7 \mathrm{pts}(7 / 28,25 \%)$.

Treatment of 28 hospitalized MM pts was variable according to various hospital COVID-19 treatment protocols. All hospitalized pts were treated by oxygen therapy; thromboembolic prophylaxis was done in all pts, too. Regarding treatments used for COVID-19, 12 pts were treated with combination of remdesivir and convalescent 
plasma, 12 pts were treated by steroids (mainly dexamethasone or methylprednisolone) and broad-spectrum antibiotics, and in 4 pts, treatment details were unknown.

Full recovery from COVID-19 infection with regression of clinic symptoms and achievement of PCR negativity of COVID-19 was observed in 19 hospitalized MM pts in median of 35 days (range $21-53$ ). Nine pts $(9 / 50,18 \%$ ) died due to severe COVID-19 pneumonia with respiratory and multi-organ failure in median of 10 days (range 7-21) from COVID-19 diagnosis. No death to thromboembolic event was observed. Mortality to COVID-19 pneumonia at group of 28 hospitalized MM pts was 32\% (9/28 pts).

The COVID-19 infection interrupted anti-myeloma treatment in all 50 pts. After recovery from COVID-19, in median 32 days, $41 \mathrm{MM}$ pts (82\%) were able to continue with previous anti-myeloma treatment. In median follow-up of 3 months from COVID-19 infection, $41 \mathrm{MM}$ pts (82\%) are alive and COVID-19 free. We recommended anti-COVID-19 vaccination according to the Czech national health guidelines in interval 3 months from COVID-19 infection for all of them.

\section{Clinical and laboratory variables and their relationship to outcome of COVID-19 infections- results of statistical analysis}

Some clinical, demographic, and laboratory parameters, such as age, gender, ISS stage, type of monoclonal immunoglobulin (MIg), comorbidities, immunoparesis, performance status according to ECOG [16], number of treatment lines, response to the last treatment line, and other, were evaluated in our cohort of $50 \mathrm{MM}$ pts with the aim to define variables important for adverse outcome of COVID-19 infection resulting in hospitalization or death. The results of statistical analysis are shown in Table 3 for COVID-19 hospitalization and in Table 4 for COVID-19 mortality.

The statistically significant parameters for COVID-19 hospitalization were as follows: responsive versus nonresponsive disease $(p=0.027)$, ECOG performance status $0-2$ versus $\geq 3(p=0.014)$, presence of comorbidities $(0-1$ versus $\geq 2, p=0.043)$. None of the other studied risk factors was significantly associated with COVID-19 hospitalization (age, gender, ISS stage, type of anti-myeloma treatment, immunoparesis, blood count values, values of creatinine and C-reactive protein).

The statistically significant parameters for COVID19 death were as follows: ECOG performance status 0-2 versus $\geq 3(p=0.001)$, presence of comorbidities $(0-1$ versus $\geq 2, p=0.007$ ), serious course of COVID-19 disease with ICU hospitalization (SU versus ICU, $p=0.001$ ), and/or invasive pulmonary ventilation treatment (IPV) $(p=0.001)$. None of the other studied risk factors was associated with COVID-19 death (age, gender, ISS stage, immunoparesis,
Table 3 Hospitalization for COVID-19 pneumonia: estimated outcome predictors based on statistical analysis with Fisher's exact test

\begin{tabular}{lll}
\hline Parameter & Comparison & $p$-value \\
\hline Gender & Female versus male & 0.559 \\
Age & $\leq 65$ versus other & 0.557 \\
& $\leq 75$ versus other & 0.548 \\
ISS stage & I+ II versus III & 0.733 \\
ECOG (performance status) & $0-2$ versus $\geq 3$ & 0.014 \\
Comorbidities & $0-1$ versus $\geq 2$ & 0.043 \\
Treatment line & $1-2$ versus $\geq 3$ & 0.104 \\
MM treatment response & Responsive versus active & 0.013 \\
& $\quad$ disease & \\
Type of last MM treatment & Daratumumab-based versus & 0.121 \\
& $\quad$ other & \\
& Lenalidomide-based versus & 0.183 \\
& other & \\
& Bortezomib-based versus & 0.239 \\
other & \\
Hemoglobin & $\leq 100$ versus other & 0.479 \\
C-reactive protein & $\leq 50$ versus other & 0.141 \\
Thrombocytes & $\leq 100$ versus other & 1.000 \\
Creatinine & $\geq 176$ versus other & 1.000 \\
Immunoparesis & Yes versus no & 0.389 \\
\hline
\end{tabular}

type of anti-myeloma treatment, COVID-19 treatment with remdesivir and convalescent plasma).

\section{Discussion}

The COVID-19 infection has affected pts globally. However, heterogeneity in outcome of COVID-19 infection has been observed to be associated with presence of comorbidities and cancer. Our retrospective study focused on outcome of 50 outpatient MM pts treated by variable types of novel drug therapy (daratumumab-based, lenalidomide-based, bortezomib-based, or other treatment), who suffered from COVID-19 infection.

The interruption of MM therapy in MM pts with symptomatic COVID-19 disease is strongly recommended [14]. In our cohort of MM pts, anti-myeloma treatment was interrupted for COVID-19 infection in all pts until full recovery from COVID-19 or death.

Optimal treatment of hospitalized MM pts with symptomatic COVID-19 disease is unclear; many pts can receive some novel agents against SARS-Cov-2 with various results [14]. In our hands, 12 hospitalized MM pts with COVID-19 pneumonia were treated with remdesivir and convalescent plasma, 9 pts fully recovered and 3 pts died; the effectivity seems to be relatively high, but our cohort is small and other clinical studies are needed. 
Table 4 Mortality for COVID-19 pneumonia: estimated outcome predictors based on statistical analysis with Fisher's exact test

\begin{tabular}{lll}
\hline Parameter & Comparison & $p$-value \\
\hline Gender & Female versus male & 0.459 \\
Age & $\leq 65$ versus other & 0.699 \\
& $\leq 75$ versus other & 0.423 \\
ISS stage & I+ II versus III & 1.000 \\
ECOG (performance status) & $0-2$ versus $\geq 3$ & 0.001 \\
Comorbidities & $0-1$ versus $\geq 2$ & 0.007 \\
Treatment line & $1-2$ versus $\geq 3$ \\
MM treatment response & Responsive versus active disease \\
Type of last MM treatment & Daratumumab-based versus other \\
& Lenalidomide-based versus other & 1.000 \\
& Bortezomib-based versus other & 0.692 \\
Hospitalization unit type & standard versus intensive care unit & 1.000 \\
Type of COVID-19 treatment & Remdesivir + convalescent plasma versus other & 1.000 \\
Immunoparesis & Yes versus no & 0.467 \\
\hline
\end{tabular}

Adult patients with hematological malignancies and COVID-19 positivity, especially hospitalized pts, have a high risk of dying [19]. Consensus paper from the European Myeloma Network prepared guidelines for therapeutic decisions in the era of the COVID-19 pandemic for MM pts [14]. Oral agent-based regimens should be considered, whereas de-intensified regimens for dexamethasone, bortezomib, carfilzomib, and daratumumab should be used based on a patient's risk and response [14]. Clinical studies have shown that MM pts under treatment with immunomodulatory drugs and proteasome inhibitors are at increased risk of severe infections [20,21]. However, some reports did not show a correlation between daratumumab or IMID-based systemic therapy and adverse COVID-19 outcome [22, 23].

Immunoparesis is associated with worse overall survival, which is not generally related to an increase risk of infections $[11,24]$. In the present cohort, we observed immunoparesis in majority of pts, but we did not see a significant correlation with poor COVID-19 outcome. Similar results have been published previously [1].

Our data suggest no significant relationship between prior lines of anti-myeloma therapy or prior type of therapy with novel drugs (daratumumab, lenalidomide, bortezomib) and outcome of COVID-19. With the limitation of our sample size, there is no clear suggestion for the need to avoid any specific MM treatment with novel drugs in indicated cases. In our hands, after median of interruption of 32 days, antimyeloma treatment was continued in $82 \%$ of pts. Importantly, our MM pts with responsive disease had superior outcome compared with those with non-responsive disease, as has been published previously [11].

In the general population, the probability of dying from COVID-19 has been reported to be between 0.5 and $3 \%$ including all COVID-19-positive patients [3]. Recent clinical trials found a higher mortality rate in hospitalized MM pts with COVID-19 (34-39\%) [25, 26], when compared to ageand sex-matched hospitalized non-MM pts with COVID-19 (16-23\%) [25, 26]. In our cohort of MM pts, mortality for all pts was $18 \%$ and for hospitalized pts $32 \%$. Serious course of COVID-19 disease with ICU hospitalization was observed in $36 \%$ pts (10/28). Thus, we confirmed previous results of higher mortality in MM pts with COVID-19.

In summary, our MM pts treated with novel drugs show a high risk of morbidity and mortality from COVID-19 infection which seems to be associated predominantly with MM diagnosis, presence of comorbidities, and performance status according to ECOG. None of the other studied risk factors was associated with poor outcome (age, gender, ISS stage, immunoparesis, type of anti-myeloma treatment). No significant correlation with various types of anti-myeloma therapies to outcome of COVID-19 infection was observed in our cohort of $50 \mathrm{MM}$ pts. The course of COVID-19 disease in MM outpatients is mostly moderate or serious with $56 \%$ of hospitalizations (28/50) and $18 \%$ of deaths (9/50).

As there is currently no specific anti-COVID treatment approved and fully successful, providing anti-COVID vaccinations to all MM pts should be strongly recommended. After recovery from COVID-19 infection, the continuation of anti-myeloma therapy is recommended.

Author contribution MK, LP, ZA, VS, MS, and ZK analyzed the data. MK and SS wrote the manuscript. All authors collected the data and approved the final manuscript.

\section{Declarations}

Conflict of interest The authors declare no competing interests. 


\section{References}

1. Susek KH, Gran Ch, Ljunggren HG et al (2020) Outcome of COVID-19 in multiple myeloma patients in relation to treatment. Eur J Hematology 105:751-754

2. Wu Z, McGoogan JM (2020) Characteristics of and important lessions from the coronarovirus disease 2019 (COVID-19) outbreak in China: summary of a report of 72314 cases the Chinese Center for Disease Control and Prevention. JAMA 312:1239-1242

3. Guan WJ, Ni ZY, Hu Yu et al (2020) Clinical characteristics of coronavirus disease 2019 in China. N Engl J Med 382:1708-1720

4. Dai M, Liu D, Liu M et al (2020) Patients with cancer appear more vulnerable for SARS-COV-2: a multicenter study during the COVID-19 outbreak. Cancer Discov 10:783-791

5. Fung M, Babik JM (2021) COVID-19 in immunocompromised hosts: what we know so far. Clin Infect Dis 72:340-350

6. Shen C, Wang Z, Zhao F et al (2020) Treatment of 5 critically ill patients with COVID-19 with convalescent plasma. JAMA 323:1582-1589

7. Grein J, Ohmagan N, Shin D et al (2020) Compassionate use of remdesivir for patients with severe COVID-19. N Engl J Med 382:2327-2336

8. Heaney JLJ, Campbell JP, Iqbal G et al (2018) Characterisation of immunoparesis in newly diagnosed myeloma and its impact on progression-free and overall survival in both old and recent myeloma trials. Leukemia 32:1727-1738

9. Chari A, Samur MK, Martinez-Lopez J et al (2020) Clinical features associated with COVID-19 outcome in multiple myeloma: first results from International Myeloma Society data set. Blood 136:3033-3040

10. Sorrig R, Klaussen TW, Salomo M et al (2017) Immunoparesis in newly diagnosed multiple myeloma patients: effects on overall survival and progression free survival in the Danish population. Plos One 12(12):e0188988

11. Heaney JLJ, Cambell JP, Iqbal G et al (2018) Characterisation of immunoparesis in newly diagnosed myeloma and its impact on progression-free and overall survival in both old and recent myeloma trials. Leukemia 32:1727-1738

12. Kazandian D (2016) Multiple myeloma epidemiology and survival: a unique malignancy. Seminar Oncol 43:676-681

13. Blinmark C, Holmberg E, Mellqvist UH et al (2015) Multiple myeloma and infections: a population-based study on $9253 \mathrm{mul}-$ tiple myeloma patients. Haematologica 100:107-113

14. Terpos E, Engelhardt M, Cook G et al (2020) Management of patients with multiple myeloma in the era of COVID-19 pandemic: a consensus paper from the European Myeloma Network (EMN). Leukemia 34:2000-2011

15. Teh BW, Harrison SJ, Pellegrini M et al (2014) Changing treatment paradigms for patients with plasma cell myeloma: impact upon immune determinants of infection. Blood Rev 28:75-86

16. Greipp PR, San Miguel J, Durie BG et al (2005) International Staging System for multiple myeloma. J Clin Oncol 23:3412-3420

17. Durie BGM, Harousseau JL, Miguel JS et al (2006) International uniform response criteria for multiple myeloma. Leukemia 20:1467-1473

18. Oken M, Creech R, Tormey D et al (1982) Toxicity and response criteria of the Eastern Cooperative Oncology Group. Am J Clin Oncol 5:649-655

19. Vijenthira A, Gorg IY, Fox TA et al (2020) Outcomes of patients with hematologic malignancies and COVID-19: a systematic review and meta-analysis of 3377 patients. Blood 136:2881-2892

20. Chen M, Zhao Y, Xu C et al (2018) Immunomodulatory drugs and the risk of serious infection in multiple myeloma: systematic review and meta-analysis of randomized and observational studies. Ann Hematol 97:925-944

21. Basler M, Lauer C, Beck U et al (2009) The proteasome inhibitor bortezomib enhances the susceptibility to viral infection. J Immunol 183:6145-6150

22. Wang B, Van Oekelen O, Mouhieddine TH et al (2020) A tertiary center experience of multiple myeloma patients with COVID-19: lessons learned and the path forward. J Hematol Oncol 13:94

23. Cook G, Ashcroft A, Pratt G et al (2020) Real-world assessment of the clinical impact of symptomatic infection with severe acute respiratory syndrome coronavirus (COVID-19 disease) in patients with multiple myeloma receiving systemic and anti-cancer therapy. Br J Haematol 190:e83-e86

24. Sorrig R, Klausen TW, Salomo M et al (2019) Risk factors for infections in newly diagnosed multiple myeloma patients: a Danish retrospective nationwide cohort study. Eur J Hematol 102:182-190

25. Martinez-Lopez J, Mateos MV, Encinas C et al (2020) Multiple myeloma and SARS-CoV-2 infection: clinical characteristics and prognostic factors of inpatient mortality. Blood Cancer J 10:103

26. Salje H, Tran KC, Lefrancq N et al (2020) Estimating the burden of SARS-Cov-2 in France. Science 369:208-211

Publisher's note Springer Nature remains neutral with regard to jurisdictional claims in published maps and institutional affiliations. 